\title{
Performance Comparison of the Kalman Filter Variants for Dynamic Mobile Localization in Urban Area Using Cellular Network
}

\author{
N. Bouzera, N. Mezhoud, A. Khireddine, and M. Oussalah
}

\begin{abstract}
A state space model for mobile terminal motion is presented which has the properties observed in true terminal motion. This model is used with a Kalman filter to combine the information of location estimates made at different times into an improved location estimate. This paper also provides experimental The performance comparison of the conventional non linear Kalman Filters and their adaptive variants for mobile dynamic location in urban area.The methodology uses TEMS Investigation software to retrieve network information including signal strength and cell-identities of various base transmitter stations (BTS). The distance from the mobile station (MS) to each BTS is therefore determined using Walfish-Ikigami radio propagation model. The different distances are therefore combined in the framework of nonlinear Kalman filter variants. In this work we compare the performance of four algorithms, based on the nonlinear Kalman Filter. For the mobile terminal localization, the results show that both of EKF, AEKF, UKF and AUKF work comparably well, in spite of the superior performance of the UKF and AUKF algorithms.
\end{abstract}

Index Terms-Mobile localization, nonlinear kalman filter variants, noise covariance adaption, cellular network.

\section{INTRODUCTION}

Localization arises repeatedly in many location-aware applications such as navigation, autonomous robotic movement, and asset tracking. Analytical localization methods include triangulation and trilateration. Triangulation uses angles, distances, and trigonometric relationships to locate an object. Trilateration, on the other hand, uses only distance measurements to identify the position of the target. Using three reference points with known locations and distances to the target object, the object can be located at the intersecting point of the three circles. However, in a dynamic system where distance measurements are noisy and fluctuate, the task of localizing becomes difficult. This uncertainty due to measurement noises renders analytical methods almost useless. Localization methods capable of accounting for and filtering out the measurement noises are desired. The method by which the distance measurements are carried out determines the sources of noise in these measurements [1]. Applications of localization GSM network appeared in the

Manuscript received May 13, 2014; revised July 25, 2014.

N. Bouzera, N. Mezhoud, and A. Khireddine are with the Genie Electric Laboratory (LGEB), Faculty of Technology, University of Bejaia, TargaOuzamour, 06000 Bejaia, Algeria (e-mail: n.bouzera@gmail.com, mezhoud.naima@gmail.com, abdelkrim.khired@gmail.com).

M. Oussalah is with the University of Birmingham, Electronics, Electrical and Computer Engineering Edgbaston, Birmingham B15 2TT (e-mail: m.oussalah@bham.ac.uk). beginning of the years 2000 because of the exponential increase of the users of the cell phone. Operators of mobile telephony were interested in the exploitation of the GSM network to ends of localization for its commercial and social benefits, for it, a service of localization appeared and used for the security of the users, in a first time, then he served to an optimal use in a second time. The quality of this service is bound closely to the precision of the positioning.

The location based services (LBS) provided in the ubiquitous environment require the accurate positions of the users and, as a result, positioning techniques have become one of the most important elements in ubiquitous networks [2]. The Global Positioning System (GPS) is the most representative method of positioning and is widely used in practical LBS systems. However, GPS cannot be utilized if line of sight visibility to the satellites is lost.

A mobile terminal, in an RF-based positioning system, measures the strengths of the signals received from at least three different fixed position stations. Then, by applying an RF propagation loss model to these signal strengths, the mobile terminal estimates its distances from the stations. By applying Kalman filter to the distances and the coordinates of the stations, the mobile terminal can estimate its position. To obtain a more accurate position from noisy distance measurements, the terminal repeats the estimation process a number of times and determines its position to be the average of the estimations. The Kalman Filter was intensively applied to dynamic systems [3]-[6]. The Kalman Filter estimates the state of a process by iteratively predicting its state and adjusting the prediction with measurements. One of the characteristics of the Kalman Filter is that it minimizes the mean of the squared error. The problem of the conventional Kalman Filter is its applicability only to linear systems, and a bad the choice of its parameters affects highly the precision of the algorithms. Other variants exist in literature to cover the nonlinear cases, and/or to tune iteratively some parameters in order to achieve higher precision.

This paper introduces a mobile GSM dynamic positioning approach using Nonlinear and/or adaptive Kalman Filter Variants. A performance comparison, in terms of accuracy, is then performed based on a real world experience Drive Tests. Section II of this paper provides a general overview of the developed system. Section III details the Kalman filter approach. Section IV details some experimental results carried out in Algiers City area.

\section{Methodologie}

The process of finding the location of the MS using the cellular network, and thereby enabling further services 
through operator server, involves several stages. The diagram in Fig. 1 shows the dynamic location process stages.

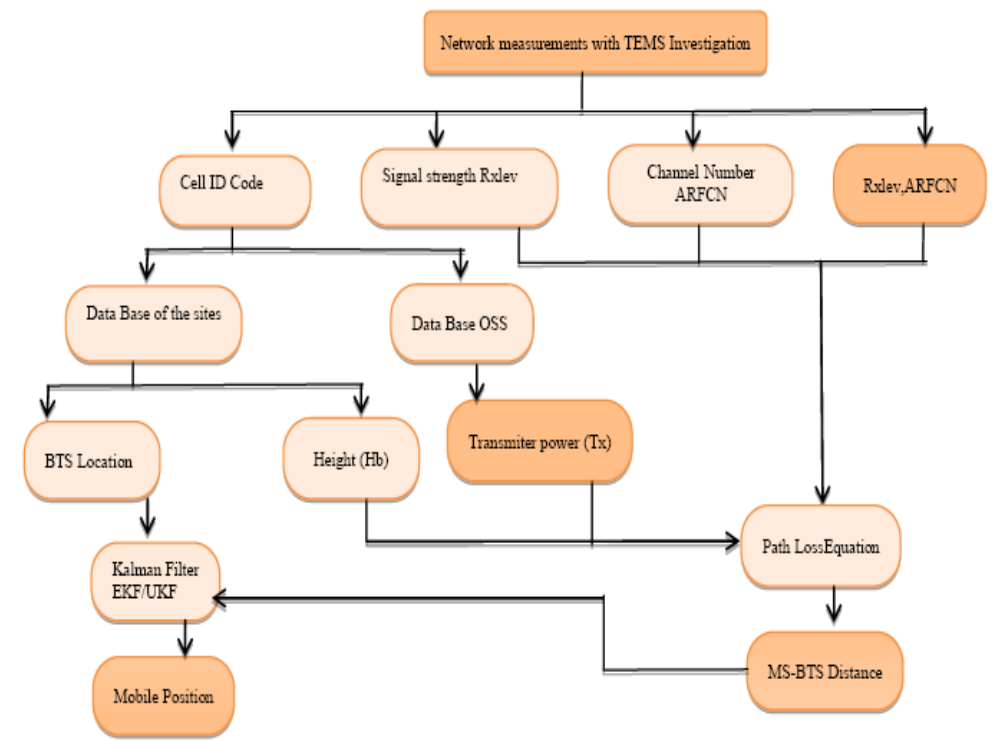

Fig. 1. Block diagram for the general methodology.

\section{A. Determination of Base Stations Locations}

The determination of the location of the BTSs is accomplished using the data base of the sites in the public operator Mobilis, which updates the location of GSM base stations throughout the Algiers. It is therefore possible to measure the latitude/longitude positioning of all the surrounding base station.

\section{B. Signal Strength Calculation Stage}

Using TEMS investigation software at the exact reference location, the strength of the signal received from the serving base station, as well as neighboring base stations, can be measured. Indeed, TEMS investigation allows determination of the Cell Identity (CI) code of each BTS communicating with MS. By forcing the hand over in order to communicate directly with a specific CI (or BTS) using the channel number of such cell as pointed by the software, TEMS investigation also allows us to display the received signal strength $(\mathrm{Rx})$ transmitted by each of the surrounding BTS pointed out at previous test.hen you submit your final version, after your paper has been accepted, prepare it in two-column format, including figures and tables.

\section{Distance Calculation}

The distance between each BTS and the handset can be determined using one of the empirical propagation models. We focused in this paper on Walfish-Ikigami propagation model [7], see [8] for an exploration of alternative models. Basically, the model provides an expressing of the path loss of the signal transmitted by the BTS $(T x)$ and received signal at the MS receiver $(R x)$, as a function of the distance between BTS and MS and the carrier frequency $f$, also determined using TEMS investigation displayed parameters.

More formally, the Walfish-Ikigamimodel defines a parameter intervening in the expression of the model are the next one, see [9] and [10].

$$
L_{\mathrm{oss}}=T x-R x: \text { Path loss }(\mathrm{dB})
$$

$f$ : Frequency bearer (MHz): $800 \leq f \leq 2000$. $h_{b}$ : Height of antenna $(\mathrm{m})$ of the base station in relation to soil: $4 \leq h_{b} \leq 50$.

$h_{m}$ : Height of antenna $(\mathrm{m})$ of the mobile station in relation to soil: $1 \leq h_{m} \leq 3$.

$h_{r}$ : Middle height $(m)$ of the buildings: $h_{r} \geq h_{m}$.

$w$ : Width of the road $(m)$ where the mobile is situated.

$b$ : Distance $(m)$ between the centers of buildings.

$d$ : Distance $(K m)$ between the BS and the mobile:

$$
0.2 \leq d \leq 5
$$

$\alpha$ : Angle (in degrees) that makes the journey with the axis of the road.

$\Delta h_{b}=h_{b}-h_{r}$ : Height of BS to the over of the roofs.

$\Delta h_{m}=h_{r}-h_{m}$ : Height of MS below the roofs.

Case of Line Of Sight LOS

$$
L p=42.64+26 \log (d)+20 \log (f)
$$

Case of Non Line Of Sight NLOS

$$
L_{o s s}= \begin{cases}L_{f s}+L_{r t s}+L_{m s d}, & \text { for urbain and suburbain } \\ L_{f}, & \text { if } L_{r t s}+L_{m s d} \leq 0\end{cases}
$$

With:

$L_{f s}$ : the attenuation in free space $(\mathrm{dB})$.

$L_{r t s}$ : the attenuation due to the diffraction on the roofs of the buildings $(\mathrm{dB})$.

$L_{m s d}$ : the attenuation due to the multiple diffractions (dB).

The attenuation in free space:

$$
L_{f s}=32,44+20 \log (d)+20 \log (f)
$$

The attenuation $(\mathrm{dB})$ due to the diffraction on the roofs of the buildings:

$$
\begin{aligned}
L_{r t s}=-16,9- & 10 \log (w)+10 \log (f)+20 \log \left(\Delta h_{m}\right) \\
& +L_{\text {ori }}
\end{aligned}
$$

$L_{\text {ori }}:$ is a term that depends on the orientation of the road in 
relation to the emitter.

$L_{\text {ori }}= \begin{cases}-10+0,3574 \alpha, & 0 \leq \alpha \leq 35^{\circ} \\ 2,5+0,075(\alpha-35), & 35^{\circ} \leq \alpha \leq 55^{\circ} \\ 4-0,1004(\alpha-55), & 55^{\circ} \leq \alpha \leq 90^{\circ}\end{cases}$

The attenuation due to the multiple diffractions:

$L_{m s d}=L_{b s h}+K_{a}+K_{d} \log (d)+K_{f} \log (f)-9 \log (b)$

$K_{a}$ and $K_{d}$ : are two factors of empiric correction of the height of the antenna.

$K_{f}$ : is a factor of adaptation of the different densities of the buildings.

With:

$$
\begin{aligned}
& L_{b s h}= \begin{cases}-18 \log \left(1+\Delta h_{b}\right), & h_{b}>h_{r} \\
0, & h_{b} \leq h_{r}\end{cases} \\
& K_{a}= \begin{cases}54, & h_{b}>h_{r} \\
54-0,8 \Delta h_{b}, & d \geq 0,5 \text { and } h_{b} \leq h_{r} \\
54-0,8 \Delta h_{b}\left(\frac{d}{0,5}\right), & d<0,5 \text { and } h_{b} \leq h_{r}\end{cases} \\
& =\left\{\begin{array}{lr}
18, & \Delta h_{b}>0 \\
18-15\left(\frac{\Delta h_{b}}{\Delta h_{m}}\right), & \Delta h_{b} \leq 0
\end{array} K_{f}\right. \\
& =\left\{\begin{array}{l}
-4+0,7\left(\frac{f}{925}-1\right), \text { average city } \\
-4+1,5\left(\frac{f}{925}-1\right), \quad \text { big city }
\end{array}\right.
\end{aligned}
$$

In the absence of detailed data on the structure of the buildings, the Cost 231 recommends the following values: $20 \mathrm{~m} \leq b \leq 50 \mathrm{~m}, w=b / 2$ [9]. In our simulation, we use the following data:

The distance (in meters) between the centers of buildings is $b=50 \mathrm{~m}$, the width of the road is $w=25 \mathrm{~m}$, the angle (in degrees) who makes the journey with the axis of the road is $\alpha=30^{\circ}$, the middle height (in meters) of the buildings is $h_{r}=15 \mathrm{~m} \mathrm{[10]}$.

The carrier frequency $f$ is determined by the following expression [11]:

$$
f=1805+0.2(\mathrm{ARFCN}-511)
$$

where ARFCN stands for BTS carrier channel number as displayed by TEMS investigation software.

Consequently, as all parameters are known, allows us to straightforwardly determine the distance $\mathrm{d}$ from the path loss $(T x-R x)$ expression.

\section{Mobile Positioning Location}

Once the distance between MS-BTS is gotten from each of the neighboring BTS, a dynamic model of Nonlinear Kalman Filters (EKF, AEK, UKF and AUKF) are used to determine the coordinates of the MS position, in terms of latitude and longitude.

\section{KALMAN FILTER}

Kalman filtering is widely known as being very useful to estimate system states that can only be observed inaccurately: it can be shown that of all possible filters, it is the one that minimizes the variance of the estimation error. This filter is not complex to implement it because its recursive nature. For a detailed description of the Kalman filter see [9] and [12].

The Kalman filter is an iterative approach that uses prior knowledge of noise characteristics to account for and filter out the noise. However, problems arise when attempting to model noise. Attempts at measuring noise are only approximations and do not indicate the real distribution of the noise. The Kalman filter can only be used for linear stochastic processes. for non-linear processes the Extended Kalman Filter (EKF) is the most widely used approach. The main concept of the EKF is the propagation of Gaussian random variables which approximates the state distribution through the first order linearization of the nonlinear model [13]. Therefore, the degree of accuracy of the EKF relies on the validity of the linear approximation and is not suitable for highly non-Gaussian conditional probability density functions, since it only updates the first two moments (mean and covariance) [13]. In addition, the calculation of the Jacobian matrix, used to linearize the nonlinear function in an EKF algorithm, can be complex causing implementation difficulties [14], [15]. In order to overcome these limitations, the Unscented Kalman Filter (UKF) has been proposed by Julier and Uhlmann [14], [15]. Based on EKF and UKF, adaptive Kalman filters have been developed to achieve much better estimation performance for non linear systems by adjusting the noise covariance matrices during estimation [16].

Once distance measurements are obtained from at least three base stations, the static model of the Kalman Filter algorithm is applied to calculate the position of the mobile phone (MS) in latitude and longitude coordinates. The accuracy of the result depends on how far it is from the location where signal strength measurements have been taken. This is determined using our GPS device at the exact location of the experiment.

Either $X_{k}\left[\begin{array}{l}L_{k} \\ l_{k}\end{array}\right]$ is the vector representing the position of the mobile station (MS) at time $k, L$ being the latitude and 1 the longitude. As the dynamic of the MS is taken into account; that is, one assumes when the MS is connected to different BTSs. In other words, the state model of the target is given by

$$
X_{k+1}=k X_{k}+W_{k}
$$

where $k$ is the state transition matrix and the system error $W_{k} \sim \mathcal{N}\left(0, Q_{k}\right)$ corresponds to the modeling error.

Either $B_{i}\left[\begin{array}{c}L_{i} \\ l_{i}\end{array}\right]$ is the vector representing the latitude and longitude of the $i$-th base station (BTS).

Let $D_{i}(k)$ be the (noised) distance between the ith BTS and the MS position at time $k ; R$ is the earth radius $(6378.135 \mathrm{~km})$. $D_{i}(k)$ is defined using the spherical law of cosines as follows:

$$
\begin{aligned}
& D_{i}(k)= \\
& R \cdot \operatorname{acos}\left[\sin \left(L_{k}\right) \sin \left(L_{i}\right)+\cos \left(L_{k}\right) \cos \left(L_{i}\right) \cos \left(l_{i-} l_{k}\right)\right]+ \\
& \varepsilon(k)
\end{aligned}
$$

where $\varepsilon(k)$ is, zero-mean Gaussian noise with the variance 
$R 0$, corresponding to the measurement model of the filter. In literature, the variance of measurement noise is noted $R$. However, in this paper, it is noted $R 0$, in order to avoid confusion with earth radius noted $R$.

\section{A. Extended Kalman Filter}

The Extended Kalman Filter (EKF) has been used for many years to estimate the state of nonlinear systems from noisy measurements, and it has been probably the first concrete application of Kalman's work on filtering [17].

The EKF algorithm is described as follows:

1) The predicting process

State updating:

$$
\hat{X}_{k}^{-}=f\left(\hat{X}_{k-1}, u_{k-1}\right)
$$

Error covariance updating:

$$
P_{k}^{-}=A_{k-1} P_{k-1} A_{k-1}^{T}+Q
$$

2) The updating process

Kalman gain determining:

$$
K_{k}=P_{k} H_{k}^{T}\left(H_{k} P_{k} H_{k}^{T}+R 0\right)^{-1}
$$

State estimation update

$$
\widehat{X}_{k}=\hat{X}_{k}^{-}+K_{k} \cdot\left(d_{i}-\widehat{D}_{i}\right)
$$

Update of state covariance:

$$
P_{k+1}=\left(I-K_{k} H_{i}\right) \cdot P_{k}
$$

where $H_{i}(k)$ stands for the Jacobian Matrix of $\widehat{D}_{i}$

$$
H_{i}(k)=\left[\frac{\partial \widehat{D}_{i}(k)}{\partial l_{k}} \frac{\partial \widehat{D}_{i}(k)}{\partial l_{k}}\right]
$$

With

$$
\begin{gathered}
\frac{\partial \widehat{D}_{i}}{\partial L_{K}}=R\left[\frac{\sin \left(L_{k}\right) \cos \left(L_{i}\right) \cos \left(l_{i}-l_{k}\right)-\cos \left(L_{k}\right) \sin \left(L_{i}\right)}{\sqrt{1-\left[\sin \left(L_{k}\right) \sin \left(L_{i}\right)+\cos \left(L_{k}\right) \cos \left(L_{i}\right) \cos \left(l_{i}-l_{k}\right)\right]^{2}}}\right] \\
\frac{\partial \widehat{D}_{i}}{\partial l_{k}}=R\left[\frac{\cos \left(L_{k}\right) \cos \left(L_{i}\right) \sin \left(l_{i}-l_{k}\right)}{\sqrt{1-\left[\sin \left(L_{k}\right) \sin \left(L_{i}\right)+\cos \left(L_{k}\right) \cos \left(L_{i}\right) \cos \left(l_{i}-l_{k}\right)\right]^{2}}}\right]
\end{gathered}
$$

\section{B. Unscented Kalman Filter}

The UKF is a recursive state estimator based on the Unscented Transform, which is a method to approximate the mean and covariance of a random variable undergoing a nonlinear transformation [18], [19]. The underlying idea is to estimate the statistics of the transformed variable from a set of $2 n+1$ points (called sigma points), with $\mathrm{n}$ being the dimension of the considered estimation problem. Sigma points are generated deterministically, on the basis of the (known) covariance matrix of the initial random variable and depending on the parameters of the filter. The procedure for implementing the UKF can be summarized as follows.

Before estimation, the state vector is initialized with the mean of $X_{0}$ and the error covariance of $P_{0}$.

$$
\hat{X}_{0}=E\left(X_{0}\right), P_{0}=E\left(\left(X_{0}-\hat{X}_{0}\right)\left(X_{0}-\hat{X}_{0}\right)^{T}\right)
$$

1) The predicting process

a) Computing sigma points: At time step $k$-1, the state estimate is assumed with mean $\hat{X}_{k-1}$ and error covariance $P_{k-1}$. A set of $2 n+1$ weighted samples called sigma points are selected as follows:

$$
\begin{gathered}
\tilde{X}_{k-1}^{(i)}=\hat{X}_{k-1}, i=0 \\
\tilde{X}_{k-1}^{(i)}=\hat{X}_{k-1}+\left(\sqrt{(n+\lambda) P_{k-1}}\right)_{i}, i=1, \ldots, n \\
\tilde{X}_{k-1}^{(i)}=\hat{X}_{k-1}-\left(\sqrt{(n+\lambda) P_{k-1}}\right)_{i}, i=n+1, \ldots, 2 n
\end{gathered}
$$

where $\lambda$ is a scaling factor determined by: $\lambda=\alpha^{2}(n+\kappa)-$ $n$. The constant $\alpha$ is set to a small positive value. It determines the spread of the sigma points around $\hat{X}_{k-1}$. The constant $\kappa$ is the secondary scaling parameter usually set to 0 .

The expression of $\left(\sqrt{(n+\lambda) P_{k-1}}\right)$, represents the $i$-th column of the matrix square root of $(n+\lambda) P_{k-1}$. Define the mean weights $W_{m}$ associated with the sigma points as

$$
W_{m}^{(i)}=\frac{\lambda}{\lambda+n}, i=0
$$

$$
W_{m}^{(i)}=\frac{\lambda}{2(\lambda+n)}, i=1, \ldots, 2 n
$$

b) Propagating sigma points: Propagate each sigma point by:

$$
\bar{X}_{k \mid k-1}^{(i)}=f\left(\tilde{X}_{k-1}^{(i)}, u_{k-1}\right)
$$

c) Determining a priori state estimate and a priori estimate error covariance.

The a priori state estimate $\hat{X}_{k \mid k-1}$ at time instant $k \mid k-1$ is computed by

$$
\hat{X}_{k \mid k-1}=\sum_{i=0}^{2 n} W_{m}^{(i)} \bar{X}_{k \mid k-1}^{(i)}
$$

To obtain the a priori estimate error covariance $P_{k \mid k-1}$, define the variance weights $W_{c}$ as

$$
\begin{gathered}
W_{c}^{(i)}=\frac{\lambda}{\lambda+n}+\left(1-\alpha^{2}+\beta\right), i=0 \\
W_{c}^{(i)}=\frac{\lambda}{2(\lambda+n)}, i=1, \ldots, 2 n \\
P_{k \mid k-1}=\sum_{i=0}^{2 n} W_{c}^{(i)}\left(\bar{X}_{k \mid k-1}^{(i)}-\hat{X}_{k \mid k-1}\right)\left(\bar{X}_{k \mid k-1}^{(i)}-\hat{X}_{k \mid k-1}\right)^{T}+ \\
Q
\end{gathered}
$$

2) The updating process

a) Computing sigma points: At time instant $k \mid k-1$, a new set of sigma points based on the a priori state estimate $\hat{X}_{k \mid k-1}$ are selected as follows:

$$
\tilde{X}_{k-1}^{(i)}=\widehat{X}_{k \backslash k-1}, i=0
$$

$\tilde{X}_{k \backslash k-1}^{(i)}=\hat{X}_{k \backslash k-1}+\left(\sqrt{(n+\lambda) P_{k \backslash k-1}}\right)_{i}, i=1, \ldots, n$ 


$$
\begin{gathered}
\tilde{X}_{k \backslash k-1}^{(i)}=\hat{X}_{k \mid k-1}-\left(\sqrt{(n+\lambda) P_{k \mid k-1}}\right)_{i}, i=n+ \\
1, \ldots, 2 n
\end{gathered}
$$

b) Computing predicted output:

Compute the corresponding output for each sigma point at time instant $k \mid k-1$ :

$$
\tilde{Y}_{k \mid k-1}^{(i)}=g\left(\tilde{X}_{k \mid k-1}^{(i)}, u_{k}\right)
$$

Then, the predicted output is determined by

$$
\widehat{Y}_{k \mid k-1}=\sum_{i=0}^{2 n} W_{m}^{(i)} \tilde{Y}_{k \mid k-1}^{(i)}
$$

c) Computing the Kalman gain $K_{k}$ :

$$
\begin{array}{r}
P_{y_{k} y_{k}}=\sum_{i=0}^{2 n} W_{c}^{(i)}\left(\tilde{y}_{k \mid k-1}^{(i)}-\widehat{y}_{k \mid k-1}\right)\left(\tilde{y}_{k \mid k-1}^{(i)}-\widehat{y}_{k \mid k-1}\right)^{T}+ \\
R 0(27) \\
P_{x_{k} y_{k}}=\sum_{i=0}^{2 n} W_{c}^{(i)}\left(\tilde{X}_{k \mid k-1}^{(i)}-\hat{X}_{k \mid k-1}\right)\left(\tilde{y}_{k \backslash k-1}^{(i)}-\hat{X}_{k \mid k-1}\right)^{T}(28) \\
K_{k}=P_{x_{k} y_{k}} P_{y_{k} y_{k}}{ }^{T}
\end{array}
$$

In (27)-(29), $P_{y_{k} y_{k}}$ is the predicted output covariance matrix. $P_{x_{k} y_{k}}$ is the cross-covariance between the predicted output and the state estimate at time instant $k \mid k-1$.

d) Determining a posteriori state estimate $\hat{X}_{k}$ and a posteriori estimate error covariance $P_{k}$ : At time step k, given the output measurement, the state estimate and error covariance are obtained by

$$
\begin{gathered}
\hat{X}_{k}=\hat{X}_{k \mid k-1}+K_{k}\left(\hat{y}_{k}-\hat{y}_{k \mid k-1}\right) \\
P_{k}=P_{k \backslash k-1}-K_{k} P_{y_{k} y_{k}} K_{k}^{T}
\end{gathered}
$$

where $\hat{y}_{k}$ represents the measurement taken at step $k$.

\section{The AEKF and AUKF Algorithms}

The process and measurement noise covariance matrices $\mathrm{Q}$ and $\mathrm{R} 0$ are assumed constant for EKF and UKF described in (3)-(7) and (12)-(31). It should be noticed that the optimality of Kalman filters estimation depends on the quality of the prior knowledge about the noise statistics [19]. Proper selection of $\mathrm{Q}$ and $\mathrm{R} 0$ is essential to the filter estimation performance. Improper $\mathrm{Q}$ and R0 may lead to large estimation error or filter divergence. To compensate for unknown or time-varying $\mathrm{Q}$ and $\mathrm{R} 0$, an adaptive approach for EKF and UKF is utilized to achieve better estimation performance and avoid divergence. The main advantage of adaptive Kalman filtering is to achieve less reliance on the prior statistical information and to adapt the noise covariance matrices according to filter learning history [20]. Therefore, building on the application of EKF and UKF, two adaptive Kalman filtering algorithms, AEKF and AUKF, are also applied in this section to obtain better tracking results. Such adaptive Kalman filters add an online adjustment block to adapt the filter parameters $\mathrm{Q}$ and R0. In each step, after achieving the state estimate, $\mathrm{Q}$ and $\mathrm{R} 0$ are updated using the Innovation based Adaptive Estimation(IAE) approach [21],

\section{for both AEKF and AUKF.}

From the incoming measurement $d(k)$ and the optimal prediction $\widehat{D}(k)$ obtained in the previous step, the innovation sequence is defined as :

$$
e(k)=d(k)-\widehat{D}(k)
$$

The covariance of $e(k)$ is written as

$$
\Phi_{e(k)}=E\left[e(k) e(k)^{T}\right]
$$

According to the maximum likelihood estimation for the multivariate normal distribution approach, the statistical sample variance of $\Phi_{e(k)}$ :

$$
\widehat{\Phi}_{e(k)}=\frac{1}{k} \sum_{i=1}^{k} e(k) e(k)^{T}
$$

The AEKF algorithm adds the following adjusting block (35) and (36) to the EKF algorithm (3)-(7)

$$
\begin{array}{r}
R 0_{k}=\hat{\phi}_{e(k)}-H_{k} P_{k} H_{k}^{T} \\
Q_{k}=K_{k} \hat{\phi}_{e(k)} K_{k}^{T}
\end{array}
$$

For the AUKF algorithm, the adjusting block (37) and (36) is added to the UKF algorithm (12)-(31)

$$
\begin{array}{r}
R 0_{k}=\hat{\phi}_{e(k)}-\sum_{i=0}^{2 n} W_{c}^{(i)}\left(\tilde{y}_{k \backslash k-1}^{(i)}-\widehat{y}_{k \mid k-1}\right)\left(\tilde{y}_{k \mid k-1}^{(i)}-\right. \\
y k \mid k-1) T
\end{array}
$$

\section{EXPERIMENT}

In order to compare the effectiveness of the four approachs, we tested the positioning of our system in the Algiers centre area. Besides, we used information obtained through drive test image as a reference to quantify the positioning error.

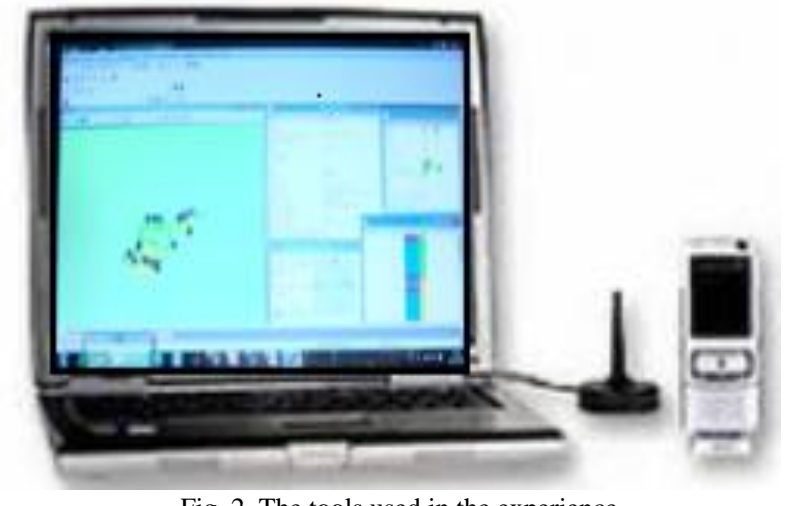

Fig. 2. The tools used in the experience.

Fig. 2 shows the drive test image of the MS positioning area. In order to extract the measurements that will be employed in the filter.

Fig. 3 provides the result in terms of signal strength as obtained using TEMS Investigation software.

Table I provides the result in terms of signal strength as obtained using TEMS Investigation software and the BTS-MS distances obtained using Walfish-Ikigami propagation model, respectively. 


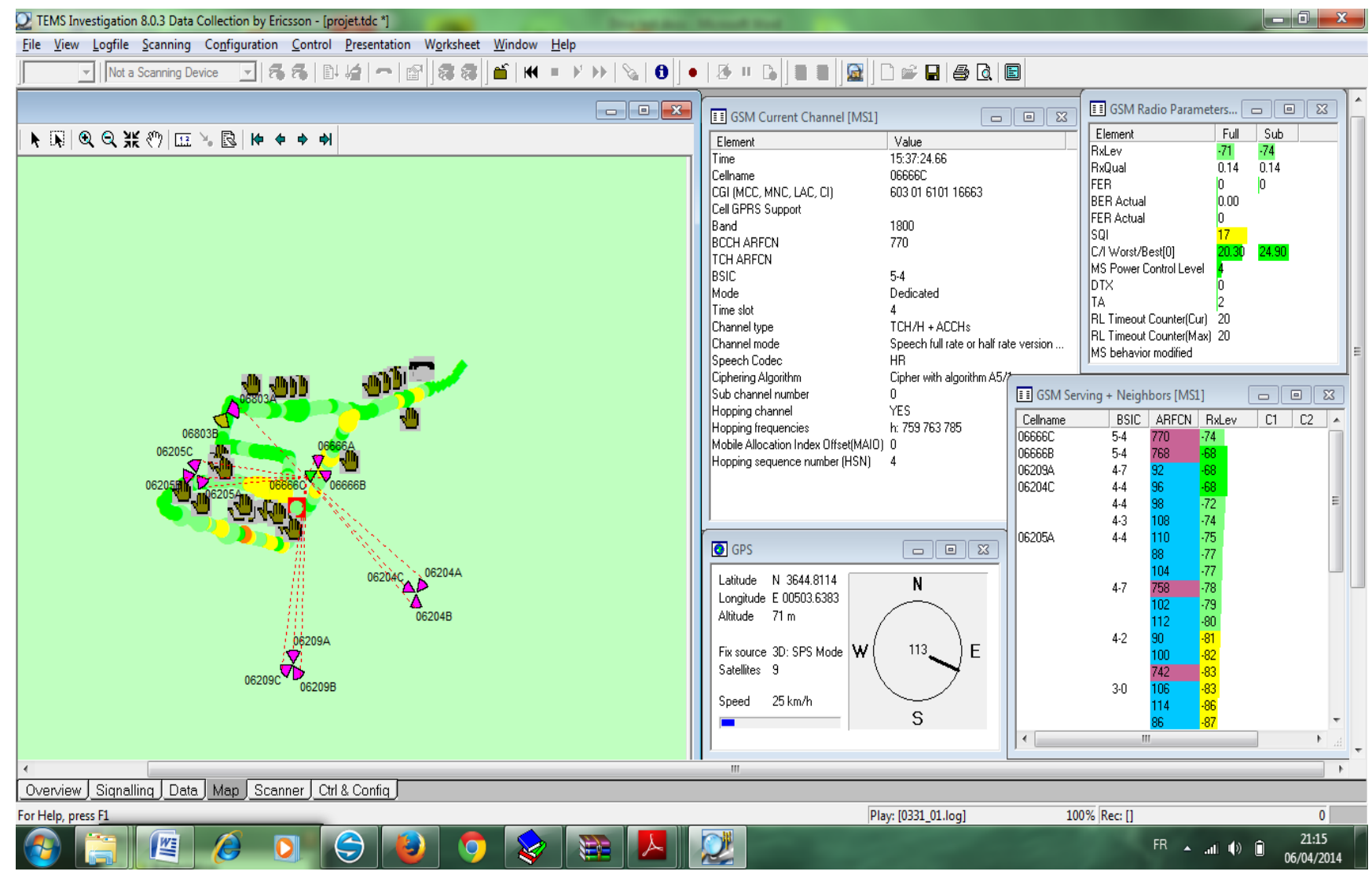

Fig. 3. Drive test experience.

TABLE I: DISTANCE CALCULATION USING WALFISH-IKIGAMI MODEL

\begin{tabular}{lllllc}
\hline \hline Cell ID & $H_{b}$ & $\mathrm{~T}_{x}$ & Rxlev & ARFCN & Walfish distances $(\mathrm{Km})$ \\
\hline $16139 \mathrm{E}$ & 52 & 43 & -69 & 764 & 0.455334 \\
$16212 \mathrm{~F}$ & 36 & 45 & -74 & 756 & 0.537776 \\
$16203 \mathrm{~F}$ & 15 & 45 & -95 & 766 & 0.443333 \\
\hline \hline
\end{tabular}

TABLE II: AVERAGE OF LOCATING ERRORS WITH DIFFERENT FILTERS

\begin{tabular}{lllll}
\hline Tracking filters & EKF & AEKF & UKF & AUKF \\
\hline $\begin{array}{l}\text { Average of locating } \\
\text { errors (Km) }\end{array}$ & 0.1789 & 0.1769 & 0.1752 & 0.1635 \\
\hline \hline
\end{tabular}

The measurements are recorded during the MS dynamics at a sampling time of 30 seconds throughout 8 minutes, which means that this test path is sampled into 16 positions. For each position, the three distances MS-BTS are calculated using Walfish-Ikigami model.

\section{Simulation Results}

To compare the performance of the presented estimation algorithms, we set the initial parameters of $P, R 0, Q$ for EKF, UKF, AEKF and AUKF to:

$$
\begin{gathered}
R 0_{0}=1 \\
P_{0}=\left[\begin{array}{cc}
0.0001 & 0 \\
0 & 0.0001
\end{array}\right] \\
Q_{0}=\left[\begin{array}{cc}
3.710^{-9} & 0 \\
0 & 4.910^{-9}
\end{array}\right] .
\end{gathered}
$$

For the UKF and AUKF algorithms additional parameters are initialized to $\kappa=0, \alpha=3, \beta=5$.

The location accuracy is the most important criterion to evaluate the localization and tracking algorithms, three metrics were used to calculate the difference between the estimated position and the real position: the Locating Error, its Cumulative Distribution Function (CDF) and the Root Mean Square Error (RMSE) calculated as follows:

$\operatorname{RMSE}(k)$

$=\sqrt{\frac{1}{N} \sum_{i=1}^{N}\left(X_{\text {real }}(k)-X_{i, \text { est }}(k)\right)^{2}+\left(Y_{\text {real }}(k)-Y_{i, \text { est }}(k)\right)^{2}}$

With $N$ is the number of BTS, in our case, $N=3 . X_{i, e s t}(k)$ and $Y_{i, e s t}(k)$ are the coordinates of the $k$-th position estimated by the $i$-th BTS. The results are illustrated in Fig. 4, Fig. 5 and Fig. 6. The average of locating errors with different filters is given in Table II.

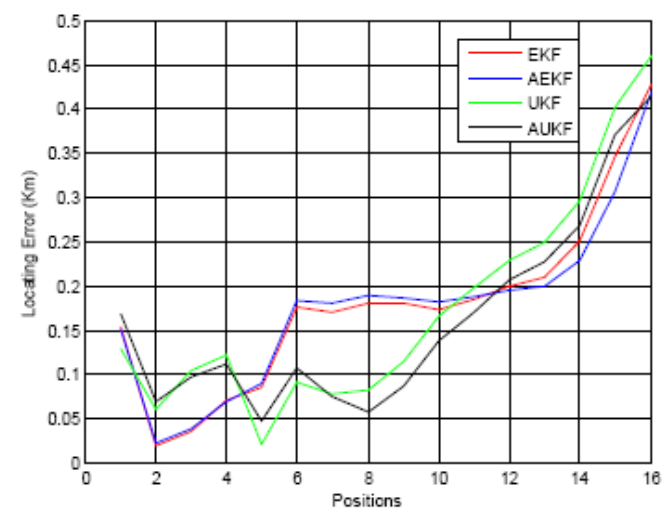

Fig. 4. Locating error at each position.

The Fig. 4, Fig. 5 and Fig. 6 show that the location error of the four algorithms are almost the same at the beginning of the tracking process, this is due to the fact that the initial 
values of the noises covariance matrices $(R 0$ and $Q)$ were well adjusted. For the rest of the trajectory, the adaptive Kalman filters (AEKF and AUKF) outperform the conventional nonlinear ones (EKF and UKF). This proves that the values of $R 0$ and $Q$ are iteratively tuned to fit the noises variations. Globally, the performance comparison of the four filters from the figures and Table II, leads to the following descent sorting according to their accuracy: AUKF, UKF, AEKF and EKF, respectively.

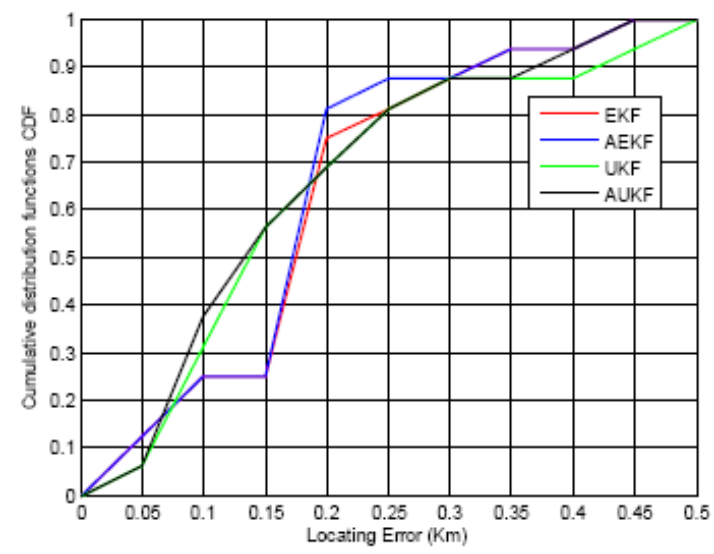

Fig. 5. Cumulative distribution Function of locating error.

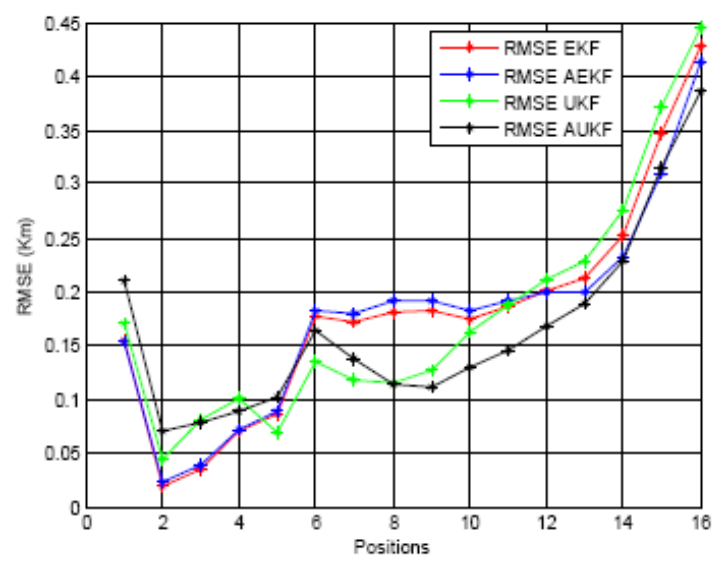

Fig. 6. RMSE of locating with different filters.

\section{CONCLUSION}

The performance comparison of the conventional non linear Kalman Filters and their adaptive variants for mobile dynamic location, has been analyzed, from the theoretical analysis and simulation results. We conclude that all of the applied filters can accomplish the dynamic location task, but the EKF has the worst accuracy, while the AUKF can achieve the best performance.

\section{REFERENCES}

[1] A. Shareef, Y.-F. Zhu, M. Musavi, and B.-X. Shen, "Comparison of MLP neural networks and kalman filter for localization in wireless sensor networks," in Proc. the 19th IASTED International Conference on Parallel and Distributed Computing and Systems, Cambridge, MA, USA, November 19-21, 2007.

[2] D. Choi, "Personalized local internet in the location-based mobile web search," Decision Support Systems, vol. 43, no. 1, pp. 31-45, 2007.

[3] R. G. Brown and P. Y. C. Hwang, Introduction to Random Signals and Applied Kalman Filtering: With Matlab Exercises and Solutions, John Wiley \& Sons, New York, NY, USA, 3rd edition, 1997.

[4] A. Gelb, Applied Optimal Estimation, The MIT Press, Cambridge, MA and London, UK, 1974.
[5] A. Kotanen, M. Hannikainen, H. Leppakoski, and T. D. Hamalainen, "Experiments on local positioning with Bluetooth," in Proc. International Conference on Information Technology: Coding and Computing [Computers and Communications] (ITCC 2003), 2003, pp. 297-303.

[6] P. S. Maybeck, Stochastic Models, Estimation, and Control, vol. I, II and III, Academic Press, 1982.

[7] S. Hamani and M. Oussalah, "Mobile location system using netmonitor and mappoint server," in Proc 6th Annual Post Graduate Symposium on the Convergence of Telecommunications, Networking and Broadcasting, Liverpool John Moores University, June 2006, pp. 17-23.

[8] V. S. Abhayawardhana, I. J. Wassell, D. Crosby, M. P. Sellars, M. G. Brown, "Comparison of empirical propagation path loss models for fixed wireless access systems," in Proc. the 61st IEEE Vehicular Technology Conference, Stockholm, Sweden, 2005, pp. 73-77.

[9] M. Shahajahan and A. Q. M. A. Hes-Shafi, "Analysis of Propagation Models for WiMAX at $3.5 \mathrm{GHz}$," M.S. thesis, Blekinge Institute of Technology, 2009.

[10] GSM: Global System for Mobile CommunicationsArchitecture, Interfaces et Identités. [Online]. Available: http://www.efort.com/r_tutoriels/GSM1_EFORT.pdf

[11] G. Gunnarsson, M. Allen, T. Rantalainen, V. Ruutu, and V.-M. Teittinen, "Location trial system for mobile phones," in Proc. the IEEE Global Telecommunication Conference. The Bridge to Global Integration, 1998, pp. 211-2216.

[12] Guvenc, C. T. Abdallah, R. Jordan, and O. Dedeoglu, "Enhancements to RSS based indoor tracking systems using kalman filters," in Proc. International Signal Processing Conference and Global Signal Processing Expo, Dallas, TX, 2003.

[13] J. L. Crassidis and J. L. Junkins, "Optimal estimation of dynamic systems, " Chapman \& Hall/CRC Applied Mathematics \& Non-linear Science Series, CRC Press, Boca Raton, FL, 2004, p. 152.

[14] S. J. Julier, J. K. Uhlmann, and H. F. Durrant-whyte, "A new method for the nonlinear transformation of means and covariances in filters and estimators," IEEE Trans. Auto. Control, vol. 45, no. 3, pp. 477-482, 2000.

[15] S. J. Julier and J. K. Uhlmann, "Unscented filtering and nonlinear estimation," Proceeding of IEEE, vol. 92, no. 3, pp. 401-422, 2004.

[16] A. Mohamed and K. Schwarz, "Adaptive Kalman filtering for INS/GPS,” Journal of Geodesy, vol. 3, pp. 193-203, 1999.

[17] M. S. Grewal and A. P. Andrews, Kalman Filtering: Theory And Practice Using MATLAB, 3rd ed. Wiley, 2008.

[18] S. J. Julier and J. K. Uhlmann, "A new extension of the Kalman filter to nonlinear systems," in Proc. AeroSense: The 11th Int. Symp. on Aerospace/Defence Sensing, Simulation and Controls, 1997, pp. $182-193$.

[19] E. Wan and R. van der Merwe, "The unscented kalman filter," in Kalman Filtering and Neural Networks, S. Haykin, Ed. Wiley, 2001, ch. 7, pp. 221-280

[20] R. Mehra, "On the identification of variances and adaptive Kalman filtering," IEEE Trans. Automatic Control, vol. 15, pp. 175-184, 1970.

[21] V. M. Moreno and A. Pigazo, Kalman Filter: Recent Advances and Applications, I-Tech, Vienna, Austria, p. 584, April 2009.

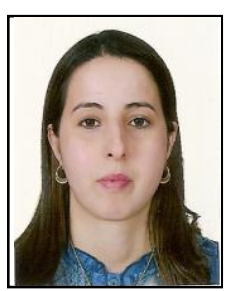

Naima Bouzera was born in Bejaia, Algeria, in 1980. She is a Ph.D. student at the Department of Genie Electric, University of A/Mira Bejaia in Algeria, where her research is the application of wireless network mobile localization with Kalman filter and its variants. She obtained her graduation state in electronic from the Bejaia University and a graduation magister in electronics in Houari Boumediene University of Science and Technology from Algeria, in 2009. She was a visiting Ph.D. student of College of Engineering and Physical Sciences in Birmingham, UK. Her present research interests include the wireless mobile localization, Kalman filter and fuzzy logic.

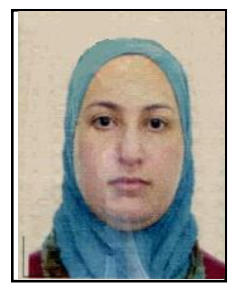

Naima Mezhoud was born in Bejaia, Algeria. She received her graduation state in electronics from Bejaia University in 2001. She also received the graduation magister in electronics systems from the Military Polytechnic School in Algiers, in 2003. In 2004, Her main research interests are in statistical signal and image processing, estimation and filtering, optimization techniques, and telecommunications. 


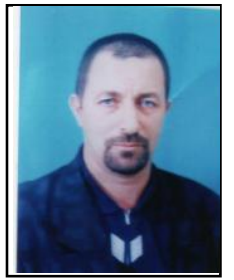

Abdelkrim Khireddine was born in Béjaia, Algeria in 1956. He received a B.S degree in mathematics in 1978 from University of Constantine, an engineering degree in telecommunication from the University of Oran with honors in 1981 and M.Sc. degree in electronics from the University of Setif in Algeria, in 1994, a Ph.D. degree in communication from the University of Setif in 2006 . He began to work at the Telecommunication Minister in 1981, and he was selected as a supervisor in 1983, and later joined the Division communication Bejaia Center. Khireddine was promoted to teach as an associated professor in 1985, in the University of Msila, then Bejaia. Pr. Khireddine was awarded a fellowship from the University of Bejaia. He was one of the founding members of the steering committee of the IEE Society Research from 2005-2007 under the number 8544624, and an official reviewer of advanced in modelling systems in the enterprise journals from 1999-2003. He was a member of the Scientific program committee member of international group of e-system research and application (TIGERA), (Tunis 2008), and was a Honorary committee member of WSEAS Conference in Romania, 2006, and MCMS'3 conference in Reggio Calabry, Italy in 2003.

As an expert in the field of telecommunication, Pr. khireddine regularly chairs international committees and sessions at national meetings, as well as organizes meetings, symposia, and workshops. He published more than 20 papers and intended more than 80 international conference, workshops, and congress. $\mathrm{He}$ as a chairman organized 3 international congress on telecommunication and applications (ICTA09, ICTA12, and ICTA14).

Recently, he is the president of Electronic Doctoral School in Bejaia University.

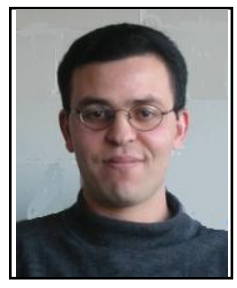

Mourad Oussalah holds a $\mathrm{PhD}$ degree in robotics and information fusion from University of Evry Vald'Essonnes in France in 1998, and he holds several academic/postdoctoral positions at University Vald'Essonnes, KU Leuven Belgium and City University of London. Since 2003 he is with University of Birmingham. He is actively working in information fusion, data mining and wireless systems where he published more than 130 international publications between conferences and journal papers and supervised more than $10 \mathrm{Ph} . \mathrm{D}$. students in the area. He is a senior member of IEEE and fellow of Royal Statistical Society. 